\title{
Assessment of Efficacy and Safety between Two Formulations of Formoterol Fumarate in Adolescents and Adults with Persistent Asthma
}

\author{
Mahesh $\mathbf{P}^{1}$, Joshi $\mathrm{J}^{2}$, Rebello $\mathrm{J}^{3 *}$, Purandare $\mathrm{SM}^{3}$ and Gogtay $\mathrm{JA}^{3}$ \\ ${ }^{1}$ Allergy, Asthma \& Chest Research Centre, Mysore, India \\ ${ }^{2}$ Department of Respiratory Medicine, BYL Nair Charitable Hospital, Mumbai, India \\ ${ }^{3}$ Clinical Research, Cipla Ltd., Mumbai, India
}

\begin{abstract}
Objective: The mandatory replacement of chlorofluorocarbons (CFCs) in pressurized metered dose inhalers (pMDIs) with non ozone depleting propellants such as hydrofluoroalkanes (HFA 134a) requires clinical testing that ensures the reformulated aerosol with HFA is as effective and well tolerated as the original CFC version. In view of this, a multicentre, randomised, parallel-group, double-blind study was conducted to compare the safety and efficacy of formoterol fumarate delivered by a MDI using the hydrofluoroalkane (HFA) 134a propellant (Cipla Ltd) with the CFC formulation (Foradil CFC pMDI, Novartis, UK) in adolescents and adults.
\end{abstract}

Methods: Patients on a stable dose of inhaled corticosteroids with a scope for improvement based on mean morning peak expiratory flow (PEF) and symptoms were randomised to receive formoterol HFA MDI 24 mg twice daily or formoterol CFC MDI $24 \mathrm{mg}$ twice daily for 12 weeks. The primary efficacy variable was the mean morning PEF and secondary variables included FEV1, symptom scores, use of relief medication and safety assessments.

Results: The difference between the treatments in the adjusted mean morning PEF (formoterol HFA-formoterol CFC) was $-4.68 \mathrm{~L} / \mathrm{min}(95 \% \mathrm{Cl}:-13.45,4.09)$. The lower limit of the $95 \%$ confidence interval was within the pre-defined limit $(20 \mathrm{~L} / \mathrm{min})$ set for non-inferiority. The results of the secondary endpoints supported the findings of the primary endpoint. The incidences of adverse events (AEs) were similar for both formulations.

Conclusion: The results of this study confirm that formoterol HFA pMDI is as effective as formoterol CFC pMDI in adolescents and adults.

Keywords: Asthma; Formoterol; HFA; CFC; Morning peak expiratory flow rate; pMDI; Non inferiority

\section{Introduction}

Although metered dose inhalers (MDIs) were introduced $>50$ years ago, they still remain the most popular and widely used inhalation devices in the treatment of lung disease, with $\sim 340$ million units used worldwide each year $[1,2]$. Although the newer dry powder inhalers offer advantages for some patients in terms of the coordination of actuation with inhalation [3], MDIs are economical to manufacture, convenient to use, and popular with patients.

For many years, chlorofluorocarbons (CFCs) have been used as medicinal aerosol propellants and solvents in MDIs owing to their nontoxic, inert and non-flammable properties. However, in accordance with the Montreal Protocol 1987 [4], which stipulates the phasing out of compounds that deplete ozone, endeavours have been made by the pharmaceutical industry to replace all CFC inhalers with alternatives, such as hydrofluoroalkanes (HFAs).

Formoterol delivered via CFC MDI is a safe and effective, long-acting beta2-agonist indicated for the treatment of asthma. It has a duration of action of $>12$ hours and protects against bronchoconstriction induced by challenge with histamine, methacholine, and exercise $[5,6]$. Any reformulation of the CFC MDI for formoterol would have to have equivalent therapeutic properties.

A CFC-free MDI formulation of formoterol has been developed by Cipla Ltd, India, which uses 1,1,1,2-tetrafluoroethane, an HFA propellant more commonly referred to as HFA-134a.

The objective of the present research programme was to compare the safety and efficacy of formoterol reformulated in HFA-propellant with the existing formoterol CFC MDI (Foradil, Novartis) at equal doses.

\section{Methods}

\section{Patient selection}

Male or female patients aged 12 years or older with a confirmed diagnosis of asthma [as defined by GINA guidelines] and a history of using inhaled corticosteroid (ICS) (either beclometasone or budesonide $(\leq 1000 \mu \mathrm{g} /$ day $)$ or fluticasone $(\leq 500 \mu \mathrm{g} /$ day $))$ for at least 4 weeks before the screening visit were included. Patients were required to have a forced expiratory volume in one second $\left(\mathrm{FEV}_{1}\right) \geq 50 \%$ and $\leq 85 \%$ of the predicted normal value when not taking short-acting bronchodilator medication, and were able to demonstrate a $\geq 15 \%$ improvement in $\mathrm{FEV}_{1}$ within 15-30 minutes after inhalation of salbutamol $(400 \mu \mathrm{g})$ unless reversibility had been documented in the previous 6 months.

All subjects were able to use the peak flow meter, to perform the required pulmonary function tests, and demonstrated correct use of the pMDI. Subjects were excluded from the study if they had received oral, depot or parenteral corticosteroids within 1 month of screening, or long acting bronchodilators (LABAs) or slow release bronchodilators within 2 weeks of screening. Subjects were also excluded if they had any clinically relevant condition that might compromise the safety of the subject or that in itself, or by its treatment, might interfere with

*Corresponding author: Juliet Rebello, Cipla Ltd, 4th floor, North Block, R\&D Centre, Vikhroli (W), Mumbai - 400 083, India, Tel: +91-22-25756420; E-mail: juliet@cipla.com

Received June 11, 2015; Accepted July 17, 2015; Published July 23, 2015

Citation: Mahesh P, Joshi J, Rebello J, Purandare SM, Gogtay JA (2015) Assessment of Efficacy and Safety between Two Formulations of Formoterol Fumarate in Adolescents and Adults with Persistent Asthma. J Pulm Respir Med 5: 278. doi:10.4172/2161-105X.1000278

Copyright: ( $) 2015$ Mahesh P, et al. This is an open-access article distributed under the terms of the Creative Commons Attribution License, which permits unrestricted use, distribution, and reproduction in any medium, provided the original author and source are credited. 
the efficacy results of the study; or had a smoking history of $\geq 10$ pack years. All women enrolled had negative pregnancy tests, and those of childbearing potential practiced acceptable methods of birth control. All subjects gave written, informed consent and were, in the opinion of the investigator, able to comply with the requirements of the protocol.

\section{Study design}

This was a randomised, double blind, multicentric, parallel-group study with a run-in period of 2-weeks, a treatment period of 12 -weeks, followed by a switch-over evaluation period of 2-weeks.

Ethics committee approval was obtained from each participating centre and the study was conducted in accordance with good clinical practice guidelines and the Declaration of Helsinki, 1996.

Eligible subjects were enrolled into the study and entered the run-in period during which they continued to take their prescribed inhaled corticosteroid (ICS) treatment. LABAs were not permitted but the short-acting $\beta_{2}$-agonist, salbutamol, was permitted as rescue medication. Subjects who were symptomatic at the end of the run-in period were then randomised to a 12-week treatment phase to either formoterol-HFA pMDI (test product) or formoterol-CFC pMDI (comparator) in a 1:1 ratio and were instructed to take 2 puffs (24 mcg) twice daily in the morning and in the evening approximately 12 hours apart during the 12 week treatment period. Since the test and comparator product devices differed in appearance, subjects also received the appropriate placebo pMDI. Subjects were followed up at $1,3,6,9$ and 12 weeks after randomisation to assess safety and efficacy.

Daily record cards were completed throughout the run-in and treatment periods. Prior to use of rescue or study medication, patients were instructed to perform three PEF measurements and record the highest value in the daily diary each morning on rising and each evening before going to bed. PEF was measured with a Mini- Wright peak flow meter (Clement Clark International Ltd, Harlow, UK). At all clinic visits, forced expiratory volume in 1 second $\left(\mathrm{FEV}_{1}\right)$ was measured using spirometry.

Each day, in addition to morning and evening PEF, the patients also recorded the number of occasions that relief medication was used during the previous day and night, and the asthma symptom scores. A patient's assessment of asthma symptoms during the day was recorded using a $0-4$ rating scale, while nighttime symptom scores were rated on a 0-3 scale, where ratings of 3 and 4 indicated very severe symptoms on the respective scales.

At the end of the run-in period, patients with scope of improvement were eligible to enter the treatment period if they fulfilled 2 of the following criteria:

- Daytime asthma score $>2$ on at least 3 of the last 7 days of the run-in period.

- $\quad$ Required salbutamol>twice daily on at least 2 days of the last 7 days of the run-in period.

- Nocturnal awakenings due to asthma on at least 2 days of the last 7 days of the run-in period.

\section{- Diurnal PEF variation $>20 \%$ on at least 2 days.}

Patients who experienced an exacerbation of their asthma during the run-in period were excluded from the study.

At the end of the 12-week treatment period, subjects who received formoterol-CFC during the treatment period were re-randomised (1:1) to receive either formoterol-HFA or to continue with formoterol-CFC to evaluate further the effects of switching from CFC to HFA. Subjects who received formoterol-HFA during the treatment period continued for another 2 weeks on formoterol-HFA.

Safety was assessed by recording of all adverse events (AEs) and serious adverse events (SAEs) and assessing changes after treatment initiation for ECG, tremor, vital signs, serum potassium and glucose levels, and clinically significant laboratory changes from end of treatment period compared with baseline values.

\section{Statistical Analysis}

The primary efficacy variable was the adjusted mean change in morning pre-dose PEF over the 12-week treatment period.

Assuming a variability for mean morning PEF of $60 \mathrm{~L} / \mathrm{min}$, a noninferiority limit of $15 \mathrm{~L} / \mathrm{min}$, a $5 \%$ significance level, $80 \%$ power and a two-sided t-test, it was estimated that the number of patients required in the per-protocol (PP) population to demonstrate non-inferiority was 115 per treatment group. To accommodate for a $20 \%$ exclusion rate from the ITT population, 138 patients were planned to be randomised per treatment group. Both the ITT and PP populations were used for analysis of the primary efficacy endpoint.

The primary efficacy variable was analysed by analysis of covariance (ANCOVA) with the baseline value prior to treatment initiation (measured as the mean of the daily values over the last seven days of the two-week run-in period), and centre as covariates.

The treatment comparison was assessed by constructing, based on ANCOVA, a two sided 95\% confidence interval (CI) for the difference between the two treatment groups (CFC as 'control' and HFA as 'test' product 'HFA-CFC'). If the lower bound of the $95 \% \mathrm{CI}$ was greater than $-20 \mathrm{~L} / \mathrm{min}$, it could be concluded that HFA was not inferior to CFC. Non inferiority was assessed based on the primary efficacy variable evaluation only.

The secondary efficacy variable FEV ${ }_{1}$ was analysed using ANCOVA with baseline and centre as covariates while for other secondary efficacy variables, the differences in the two treatment groups for total symptom scores, intake of rescue medication were tested by nonparametric tests. AEs, SAEs and discontinuations due to AEs were reported descriptively. All other safety data was collated and summarised by treatment group.

\section{Results}

Three hundred and twenty four patients were recruited into the study. Of these, 44 patients were withdrawn prior to randomisation. The most common reasons for withdrawal were patients not fulfilling entry criteria $(n=18)$ or not fulfilling the randomization criteria $(n=26)$.

Of the 280 subjects who were randomised and received at least one dose of the study medication (HFA group $n=141$, CFC group $n=139$ ), 2 subjects were withdrawn (lost to follow-up) after randomization in the HFA group and 1 subject in the CFC group. Therefore, 277 subjects were included in the ITT population (139 subjects in the HFA group and 138 subjects in the CFC group). Of the 277 subjects in the ITT population, 33 subjects were excluded from the PP population (Table 1).

Demography and baseline characteristics were well matched between the two treatment groups (Table 2). Both the treatment groups had populations with comparable baseline lung function (PEF, percentage predicted $\mathrm{FEV}_{1}$ and $\mathrm{FEV}_{1}$ ). The mean $\%$ predicted $\mathrm{FEV}_{1}$ was approximately

$68 \%$ and $66 \%$ in HFA and CFC groups respectively. At baseline, 


\begin{tabular}{|l|c|c|}
\hline & Formoterol HFA & Formoterol CFC \\
\hline Randomised & 141 & 139 \\
\hline $\begin{array}{l}\text { Withdrawn immediately after } \\
\text { randomization (lost to follow up) }\end{array}$ & 2 & 1 \\
\hline Included in ITT population & 139 & 138 \\
\hline Completed & 123 & 129 \\
\hline Included in PP population & 120 & 124 \\
\hline Excluded from PP population & 19 & 14 \\
\hline Major protocol violations & 7 & 7 \\
\hline Adverse events & 2 & 2 \\
\hline Lost to follow up & 9 & 5 \\
\hline Withdrawal of consent & 1 & 0 \\
\hline
\end{tabular}

Table 1: Patient disposition.

\begin{tabular}{|l|c|c|}
\hline & Formoterol HFA & Formoterol CFC \\
\hline Sex, Males/Females & $73 / 66$ & $62 / 76$ \\
\hline Age, years & 38.2 & 39.1 \\
\hline Mean & $15.2-64.3$ & $12.1-65.8$ \\
\hline Range & $1.87(0.54)$ & $1.74(0.52)$ \\
\hline FEV 1 (L) Mean(SD) & $68.1(11.9)$ & $66.1(13.3)$ \\
\hline FEV 1 (\% predicted) Mean(SD) & $270(82.8)$ & $259(72.9)$ \\
\hline PEF $($ L/min) Mean (SD) & $267(83.0)$ & $257(75.2)$ \\
\hline Morning & 1.56 & 1.54 \\
\hline Evening & 1.95 & 1.73 \\
\hline $\begin{array}{l}\text { Salbutamol use, puffs/night } \\
\text { (Median) }\end{array}$ & 27.3 & 27.1 \\
\hline $\begin{array}{l}\text { Salbutamol use, puffs/day } \\
\text { (Median) }\end{array}$ & 29.5 & 30.2 \\
\hline$\%$ of daytime asthma scores $=0 *$ & * of nighttime asthma & \\
\hline scores=0**
\end{tabular}

*Unless otherwise expressed, values presented as means (SD); * $0=$ very well, no symptoms; ** $0=$ no symptoms, slept through the night

Table 2: Characteristics and asthma profile of efficacy population at baseline.

both treatment groups showed similar baseline symptom scores and use of relief medication.

\section{Mean morning PEF}

Mean morning PEF values derived from diary card readings were analysed for both PP and ITT populations. In both the populations, a clinically relevant increase in mean morning PEF at week 12 was observed in both formoterol HFA and formoterol CFC treatment groups (Figure 1).

In the ITT population, the overall improvement in mean morning PEF, adjusting for centre and baseline was 26.3 and $21.6 \mathrm{~L} / \mathrm{min}$ in the formoterol HFA and formoterol CFC groups, respectively (Table 3). The estimated treatment difference (formoterol HFA-formoterol CFC) was $-4.68 \mathrm{~L} / \mathrm{min}$ and the $95 \%$ confidence interval was $(-13.45,4.09)$. The lower CI limit was within the pre-defined non-inferiority limit of $20 \mathrm{~L} / \mathrm{min}$, indicating that the HFA formulation is at least as effective as the CFC formulation.

For the PP population, the $95 \%$ confidence interval was $-12.93,4.94$ which further confirmed noninferiority of the HFA formulation.

\section{Clinic FEV 1}

No significant difference in clinic $\mathrm{FEV}_{1}$ at endpoint was observed between formoterol HFA and formoterol CFC in both the ITT and PP populations (Table 4). However, the mean $\mathrm{FEV}_{1}$ at baseline in the ITT population was slightly higher in the HFA group $(1.87 \mathrm{~L})$ compared with the CFC group $(1.74 \mathrm{~L})$. The difference was not statistically significant at baseline or at Week 1. However, at Weeks 3, 6, 9 and 12 , the mean $\mathrm{FEV}_{1}$ was statistically significantly higher $(\mathrm{p}<0.05)$ in the HFA group compared with the CFC group.

\section{Symptom scores and use of relief medication}

Median daytime and nighttime symptom scores as well as daily use of relief medication were reduced by both formulations (Figure 2 and Table 5).

\section{Switch-evaluation data}

Following a further two weeks treatment, the morning PEF, evening PEF and diurnal variation were comparable between treatments indicating no deterioration in respiratory parameters after switching from CFC to HFA.

\section{Safety}

Overall, both treatment formulations were well tolerated and had comparable AE profiles (Table 6).

The incidence of AEs was similar in the two groups. The most common AEs during the treatment period were headache followed by pyrexia. Four SAEs occurred during the study, 2 SAEs in each treatment group, none of which was related to study medication. No clinically significant changes from baseline were found in vital-sign measurements, ECG, tremor assessment or laboratory tests in either group throughout the study.

\section{Conclusions}

For the primary efficacy variable (morning PEF) as the two sided 95\% CI for the difference between the two treatment groups lies well above the proposed non-inferiority limit of $-20 \mathrm{~L} / \mathrm{min}$, it can be concluded that the formoterol-HFA pMDI is clinically non-inferior to the formoterol-CFC pMDI. This conclusion is supported by the secondary efficacy variables. There was no reduction in morning or evening PEF after a two week switch from CFC to HFA. The nature and frequency of AEs in both groups were similar.

\section{Discussion}

This study suggests that substantial clinical efficacy may be achieved with formoterol at a dose of $24 \mathrm{mcg}$ twice daily when administered via an MDI using either a CFC or HFA propellant. Furthermore, the magnitude of the clinical improvement from baseline observed with

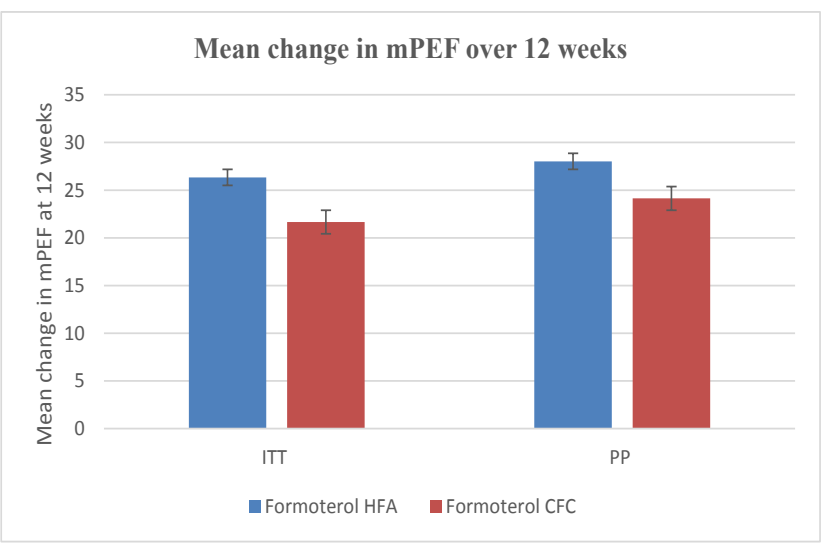

Figure 1: Mean change in MPEF at week 12 (ITT and PP populations). 
Citation: Mahesh P, Joshi J, Rebello J, Purandare SM, Gogtay JA (2015) Assessment of Efficacy and Safety between Two Formulations of Formoterol Fumarate in Adolescents and Adults with Persistent Asthma. J Pulm Respir Med 5: 278. doi:10.4172/2161-105X.1000278

Page 4 of 4

\begin{tabular}{|c|c|c|c|c|}
\hline Lung function parameter & Treatment & $\mathbf{N}$ & Mean (SD) morning PEF during treatment & $\begin{array}{c}\text { Adjusted mean change } \\
\text { from baseline }\end{array}$ \\
\hline mPEF (L/min)-ITT & Formoterol HFA & 139 & $295(84.4)$ & 26.34 \\
\hline & Formoterol CFC & 138 & $281(73.1)$ & 21.66 \\
\hline mPEF (L/min)-PP & Formoterol HFA & 120 & $301(84.5)$ & 28.02 \\
\hline & Formoterol CFC & 124 & $286(69.3)$ & 24.14 \\
\hline
\end{tabular}

Table 3: Statistical analyses of mPEF for formoterol HFA pMDI versus formoterol CFC pMDI.

\begin{tabular}{|c|c|c|}
\hline & Formoterol HFA & Formoterol CFC \\
\hline FEV $_{\mathbf{1}}$ (L) Mean ( SD) & $\mathbf{N}=138$ & $\mathbf{N}=138$ \\
\hline Week 1 & $1.89(0.57)$ & $1.75(0.54)$ \\
\hline Week 3* $^{*}$ & $1.92(0.60)$ & $1.79(0.52)$ \\
\hline Week 6 $^{*}$ & $1.90(0.61)$ & $1.77(0.51)$ \\
\hline Week 9*$^{*}$ & $1.93(0.59)$ & $1.78(0.51)$ \\
\hline Week 12* & $1.87(0.61)$ & $1.75(0.53)$ \\
\hline
\end{tabular}

${ }^{*} \mathrm{p}<0.05$ formoterol HFA versus formoterol CFC

Table 4: Mean FEV1 over 12 weeks (Formoterol HFA versus Formoterol CFC).
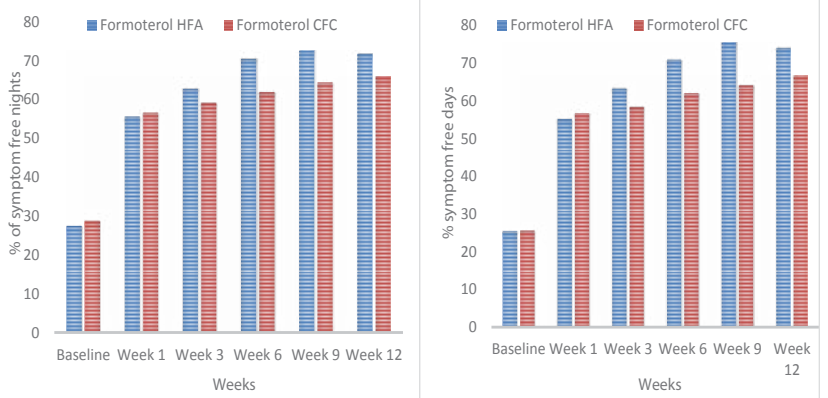

Figure 2: Percentage of symptom free days and nights (Formoterol HFA versus Formoterol CFC).

both treatments was therapeutically similar, as evidenced by statistical non-inferiority of the primary efficacy parameter assessed.

The change in mean morning PEF, was deemed clinically meaningful. Furthermore, no statistically significant differences between the two formulations were observed in mean morning PEF, in either population, as the lower limits of the $95 \%$ confidence intervals were above the pre-defined $20 \mathrm{~L} / \mathrm{min}$ non-inferiority limit. These results indicate comparable efficacy between the HFA and CFC formulations of formoterol.

Marked improvements from baseline in $\mathrm{FEV}_{1}$, daytime and nighttime symptom scores and daily use of relief medication were observed with both treatments, further illustrating the efficacy of formoterol in the treatment of asthma.

The baseline and eligibility characteristics of the population under study showed that there was room for clinical improvement of lung function and asthma symptoms in response to study medication, hence avoiding a conclusion of non inferiority through nonresponse.

Additionally, the observed mean improvements in the clinical parameters evaluated for the primary endpoint $\mathrm{mPEF}$ were consistent between the ITT and PP populations, thereby confirming the validity of the study results. The two formulations of formoterol were well tolerated and demonstrated comparable tolerability profiles, with the formulations exerting similar incidences of AEs, which were not unexpected for the populations under investigation.

The operation of a MDI is independent of a patient's diagnosis, and

\begin{tabular}{|c|c|c|}
\hline & Formoterol HFA & Formoterol CFC \\
\hline \multicolumn{3}{|c|}{ Nighttime use of rescue } \\
\hline Baseline & 1.56 & 1.54 \\
\hline 12 weeks & 0.10 & 0.19 \\
\hline \multicolumn{3}{|c|}{ Daytime use of rescue } \\
\hline Baseline & 1.95 & 1.73 \\
\hline 12 weeks & 0.05 & 0.15 \\
\hline
\end{tabular}

Table 5: Mean number of puffs of rescue medication usage at baseline and at 12 weeks.

\begin{tabular}{|c|c|c|}
\hline & Formoterol HFA & Formoterol CFC \\
\hline Pyrexia & $13(1.42)$ & $30(3.60)$ \\
\hline Rhinitis & $17(4.96)$ & $17(3.60)$ \\
\hline Rhinitis Allergic & $13(2.13)$ & $18(2.16)$ \\
\hline Headache & $29(7.80)$ & $25(9.35)$ \\
\hline
\end{tabular}

Table 6: Frequency (\%) of most commonly reported (>2\%) adverse events.

the pharmacological mechanism of formoterol is the same for asthma and COPD. While comparable efficacy of the HFA formulation and the CFC formulation of formoterol was demonstrated in asthmatic populations in this study, there is no reason to anticipate that the effects of the formoterol HFA inhaler will differ from those of the CFC inhaler in terms of bronchodilator efficacy in patients with COPD. This is because other drug classes, redeveloped from CFC- to HFA-containing inhalers, have shown similar clinical benefits in COPD.

The comparable efficacy and tolerability profiles of the formoterol HFA and formoterol CFC formulations, therefore, facilitate a seamless transition to the new CFC-free inhalers.

In summary, this study has shown that in adolescents and adults with persistent asthma may be switched from the formoterol CFC MDI (Foradil) to the formoterol HFA MDI (Cipla Ltd) with maintenance of good clinical efficacy and tolerability profiles.

\section{References}

1. Partridge MR (1994) Metered-dose inhalers and CFCs: what respiratory physicians need to know. Respir Med 88: 645-647.

2. Woodcock A (1995) Continuing patient care with metered-dose inhalers. Aerosol Med 8 Suppl 2: S5-10.

3. van Beerendonk I, Mesters I, Mudde AN, Tan TD (1998) Assessment of the inhalation technique in outpatients with asthma or chronic obstructive pulmonary disease using a metered-dose inhaler or dry powder device. J Asthma 35: 273-279.

4. Montreal protocol on substances that deplete the ozone layer (1987) Int Leg Mater 16: 1541.

5. Derom EY, Pauwels RA (1992) Time course of bronchodilating effect of inhaled formoterol, a potent and long acting sympathomimetic. Thorax 47: 30-33.

6. Bartow RA, Brogden RN (1998) Formoterol. An update of its pharmacological properties and therapeutic efficacy in the management of asthma. Drugs 55 303-322. 